\title{
ROTINA COMPUTACIONAL PARA A PREVISÃO DA CAPACIDADE DE CARGA EM ESTACAS
}

\section{Computational routine to provision of the capacity of load in piles.}

\author{
Tobias Ribeiro Ferreira ${ }^{1}$, Rodrigo Gustavo Delalibera ${ }^{2}$, Wellington Andrade da Silva ${ }^{3}$ \\ Recebido em 16 de fevereiro de 2014; recebido para revisão em 28 de abril de 2014; aceito em 08 de maio de \\ 2014; disponível on-line em 06 de junho de 2014.
}

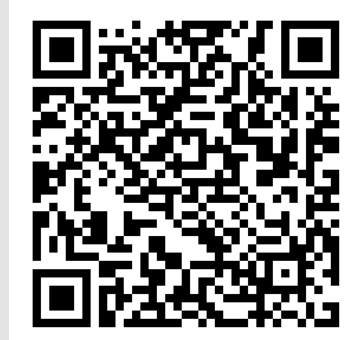

PALAVRAS CHAVE:

Capacidade de carga;

Fundações por estacas;

Planilhas eletrônicas.

\footnotetext{
* Contato com o autor:
}

${ }^{1}$ e-mail : tobias.trf@hotmail.com

Professor Substituto, Universidade Federal de Goiás - Campus Catalão, Departamento de Engenharia Civil

2e-mail : rodrigo_delalibera@ufg.br (R. G. Delalibera )

Professor Adjunto III, Universidade Federal de Goiás - Campus Catalão, Departamento de Engenharia Civil

${ }^{3}$ e-mail : wellington_andrade@ufg.br (W.A.da Silva )

Professor Adjunto I, Universidade Federal de Goiás - Campus Catalão, Departamento de Engenharia Civil

\section{INTRODUÇÃO}

As edificações em geral, são sujeitas às ações horizontais (vento e desaprumo), ações gravitacionais permanentes, ações gravitacionais acidentais, efeitos de temperatura, em alguns casos ações excepcionais como sismos e choques. Os esforços internos provocados por estas ações produzem reações nos apoios que são transmitidos ao solo por meio dos elementos de fundação, sejam elas superficiais ou profundos. Quando o solo não apresenta condições de resistência nas camadas superficiais (até uma profundidade de dois metros) faz-se necessário que as fundações alcancem camadas profundas e resistentes, e neste caso, podem ser utilizados tubulões ou estacas, as quais são definidas pela NBR 6118 (ABNT, 2010) como elementos de 
fundação que transmitem as cargas ao terreno pela base (resistência de ponta), por sua superfície lateral (resistência de atrito do fuste) ou por uma combinação das duas.

As estacas disponíveis no mercado são classificadas em duas macrocategorias: estacas de deslocamento que são introduzidas no terreno por meio de processo que não provoca a retirada do solo e estacas escavadas, que são aquelas executadas "in situ" por meio de perfuração manual ou mecânica do terreno, com remoção de material com ou sem a utilização de fluido estabilizante (lama bentonitica), utilizando ou não revestimento para proteção do fuste.

No dimensionamento da fundação é necessário saber a capacidade resistente das estacas e qual o nível de solicitação que os solos resistem sem ruptura ou deformação excessiva.

A determinação correta da capacidade de carga de uma fundação é a base para o desenvolvimento de um projeto que seja seguro e economicamente viável. Atualmente existem vários programas que fazem esses cálculos, contudo em sua maioria são programas que utilizam apenas um único método. $O$ desenvolvimento dessa pesquisa foi justificado pela possibilidade que um engenheiro, estudante, ou pesquisador terá para avaliar a melhor metodologia de cálculo, bem como o tipo de execução e ainda as possibilidades para os mais variados tipos de fundações em estacas, utilizando uma rotina computacional de fácil manuseio e gratuita.

\section{OBJETIVO}

Este artigo tem por objeto a apresentação de uma rotina computacional para o cálculo de capacidade de carga geotécnica em estacas, como também, comparar os resultados teóricos com resultados obtidos por meio de provas de carga.

\section{METODOLOGIA}

Desenvolveu-se uma r rotina
computacional utilizando-se em planilhas
eletrônicas com implementação de macros e
linguagem de programação VBA. Por meio da rotina

computacional, o usuário terá condições de estimar a capacidade geotécnica de estacas, inserindo de dados geotécnicos, tipos de estacas e característicos do solo.

\subsection{MÉTODOS DE CÁLCULO}

$\mathrm{Na}$ definição da capacidade de carga para estacas, a primeira etapa é calcular sua resistência estrutural. Essa resistência é calculada com base nas dimensões e no material da estaca. Para as estacas pré-moldadas, esse valor pode ser obtido por meio da aplicação das hipóteses básicas do concreto armado e protendido. Tais valores estão descritos nos manuais dos fabricantes ou em tabelas de capacidade de carga estrutural, como pode ser observado na Tabela 1.

$\mathrm{Na}$ Tabela 1 observa-se que a capacidade de carga estrutural aumenta à medida que o diâmetro aumenta e a tensão de trabalho poderá variar para uma mesma estaca.

Uma vez definida a capacidade estrutural das estacas é preciso entender que o sistema estaca-solo submetido a uma carga vertical, resiste às solicitações por meio da resistência ao cisalhamento gerada ao longo de seu fuste e pelas tensões normais geradas ao nível de sua ponta. A carga que leva a ruptura desse conjunto é denominada de capacidade de carga. Carga essa que pode ser avaliada por meio de métodos estáticos, dinâmicos ou provas de carga. Os métodos estáticos se dividem em métodos racionais ou teóricos (aqueles que utilizam soluções teóricas de capacidade de carga e parâmetros do solo) e métodos semiempíricos (aqueles que se baseiam em ensaios "in situ" de penetração, como por exemplo, o SPT e o CPT).

Há também os métodos empíricos, a partir dos quais se pode estimar de forma aproximada a capacidade de carga de estaca com base na descrição das camadas do solo ao longo do fuste e da experiência do profissional.

A rotina computacional desenvolvida nesta pesquisa utiliza os métodos semiempíricos, dentre os quais foram utilizados as metodologias propostas por Aoki-Velloso (1975), DécourtQuaresma (1978) e Teixeira (1996). 
TABELA 1: Capacidade de carga estrutural de estacas pré-moldadas de concreto.

\begin{tabular}{|c|c|c|c|c|}
\hline Tipo de estaca & $\begin{array}{c}\text { Dimensão } \\
(\mathrm{cm})\end{array}$ & $\begin{array}{l}\text { Carga usual } \\
\text { (kN) }\end{array}$ & $\begin{array}{c}\text { Carga máxima } \\
(\mathrm{kN})\end{array}$ & Observação \\
\hline \multirow{4}{*}{$\begin{array}{c}\text { Pré-moldada vibrada } \\
\text { quadrada } \\
\text { ( } \sigma=6 \text { à } 10 \mathrm{MPa})\end{array}$} & $20 \times 20$ & 300 & 400 & \multirow{4}{*}{$\begin{array}{l}\text { Disponíveis até } 8 \text { metros; } \\
\text { Podem ser emendadas. }\end{array}$} \\
\hline & $25 \times 25$ & 450 & 600 & \\
\hline & $30 \times 30$ & 600 & 900 & \\
\hline & $35 \times 35$ & 900 & 1200 & \\
\hline \multirow{3}{*}{$\begin{array}{l}\text { Pré-moldada vibrada } \\
\text { circular } \\
\text { ( } \sigma=9 \text { à } 11 \mathrm{MPa})\end{array}$} & $\varnothing 22$ & 300 & 400 & \multirow{3}{*}{$\begin{array}{l}\text { Disponíveis até } 10 \text { metros; } \\
\text { Podem ser emendadas; } \\
\text { Podem ter furo central }\end{array}$} \\
\hline & $\varnothing 29$ & 500 & 600 & \\
\hline & $\varnothing 33$ & 700 & 800 & \\
\hline \multirow{3}{*}{$\begin{array}{l}\text { Pré-moldada } \\
\text { protendida circular } \\
\text { ( } \sigma=10 \text { à } 14 \mathrm{MPa})\end{array}$} & $\varnothing 20$ & 300 & 350 & \multirow{3}{*}{$\begin{array}{l}\text { Disponíveis até } 12 \text { metros; } \\
\text { Podem ser emendadas; } \\
\text { Podem ter furo central }\end{array}$} \\
\hline & $\varnothing 25$ & 500 & 600 & \\
\hline & $\varnothing 33$ & 800 & 900 & \\
\hline \multirow{6}{*}{$\begin{array}{l}\text { Pré-moldada } \\
\text { centrifugada } \\
\text { ( } \sigma=9 \text { à } 11 \mathrm{MPa})\end{array}$} & $\varnothing 20$ & 250 & 300 & \multirow{6}{*}{$\begin{array}{l}\text { Disponíveis até } 12 \text { metros; } \\
\text { Podem ser emendadas; } \\
\text { Com furo central (ocas) e } \\
\text { paredes de } 6 \text { à } 12 \mathrm{~cm} \text {. }\end{array}$} \\
\hline & $\varnothing 26$ & 400 & 500 & \\
\hline & $\varnothing 33$ & 600 & 750 & \\
\hline & $\varnothing 42$ & 900 & 1150 & \\
\hline & $\varnothing 50$ & 1300 & 1700 & \\
\hline & $\varnothing 60$ & 1700 & 2300 & \\
\hline
\end{tabular}

$\sigma=$ tensão de trabalho (função da resistência ao escoamento das barras de aço e da resistência característica do concreto à compressão) FONTE: Velloso e Lopes (2002).

Pode-se dizer que as metodologias foram desenvolvidas a partir da definição de ALONSO (1983), onde é descrito uma estaca como sendo um elemento estrutural esbelto que, colocado no solo por cravação ou perfuração, tem a finalidade de transmitir ações ao mesmo. Diz ainda, que estes elementos apresentam capacidade de dissipar os esforços solicitantes em função da resistência sob sua extremidade inferior, pela resistência ao longo do fuste por meio do atrito lateral, ou ainda pela combinação das duas. A Figura 1 mostra esquematicamente como é considerada cada parcela de resistência. 


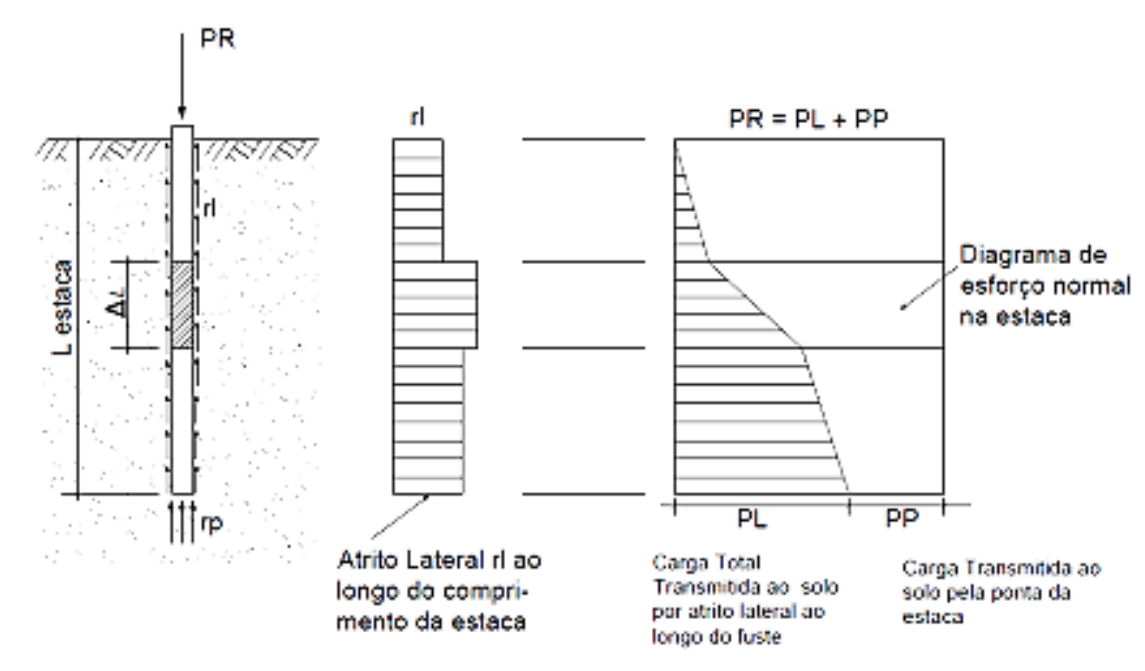

FIGURA 1: Mecanismo de Resistência da Fundação por Estaca

FONTE: Cintra e Aoki (2010).

Com base na Figura 1 a capacidade de carga de uma estaca é desenvolvida da seguinte forma:

$$
R=R_{L}+R_{p} \quad \text { Eq.[01] }
$$

Em que:

$\mathrm{R}$ : Capacidade de carga;

$\mathrm{R}_{\mathrm{L}}$ : Parcela de resistência lateral;

$\mathrm{R}_{\mathrm{p}}$ : Parcela de resistência de ponta.

A Equação 1 é a origem para todos os métodos utilizados neste trabalho, a diferença entre eles se dará pelas considerações e coeficientes sugeridos por cada um dos autores.

\subsubsection{Método de cálculo de Aoki-Velloso}

Aoki e Velloso (1975) propõem critérios para a determinação da resistência de ponta $\left(R_{p}\right)$ e da resistência lateral $\left(R_{l}\right)$ que compõem a capacidade de carga da estaca (R). As Equações 2 e 3 apresentam as parcelas da resistência de ponta e da resistência lateral.

$$
\begin{gathered}
R_{p}=r_{p} \cdot A_{p} \\
R_{L}=U \cdot \sum\left(r_{i} \cdot \Delta_{L}\right)
\end{gathered}
$$

Em que:

$r_{p}$ é a capacidade de carga do solo na cota de apoio do elemento estrutural de fundação (MPa);

$A_{p}$ é a área da seção transversal da ponta $\left(\mathrm{m}^{2}\right)$; $\mathrm{r}_{\mathrm{i}}$, é a tensão media de adesão ou de atrito lateral na camada de espessura $\Delta_{\mathrm{L}}(\mathrm{MPa})$;

U é o perímetro da seção transversal do fuste (m).
As parcelas $r_{p}$ e $r_{i}$ são parâmetros geotécnicos que foram inicialmente obtidos, por relações dos ensaios de penetração estática (CPT), onde o valor de resistência de ponta do cone $\left(q_{c}\right)$ é relacionado com a resistência de ponta da estaca, e $o$ atrito lateral unitário na luva $\left(\mathrm{f}_{\mathrm{s}}\right)$ relacionado com a resistência produzida ao longo do fuste, como apresentado nas Equações 4 e 5.

$$
\begin{gathered}
r_{p}=\frac{q_{c}}{F_{1}} \\
r_{L}=K \cdot N_{S P T}
\end{gathered}
$$

No meio técnico brasileiro, entretanto, como a ocorrência de ensaios tipo CPT é rara e costumeiramente utiliza-se o ensaio SPT (Standard Penetration test), foram criados parâmetros de correlações para a resistência de ponta e atrito. Essas correlações podem ser realizadas por meio da Equação 6.

$$
q_{c}=K \cdot N_{S P T}
$$

Sendo que o coeficiente $K$ é um valor dependente do tipo de solo. Esse recurso permite que o atrito lateral seja expresso em função do $\mathrm{N}_{\mathrm{SPT}}$, utilizando simplesmente a razão de atrito $(\alpha)$ da Equação 7.

$$
\alpha=\frac{f_{s}}{q_{c}}
$$

Com isso o valor da resistência de atrito lateral passa a ser expresso pela Equação 8. 


$$
f_{s}=\alpha \cdot q_{c}=\alpha \cdot K \cdot N_{S P T} \quad \text { Eq. [08] }
$$

Sendo o coeficiente $\alpha$ função do tipo de solo.

Com essas correlações entre os ensaios, as Equações 4 e 5 foram reescritas obtendo-se as equações 9 e 10.

$$
\begin{aligned}
& r_{p}=\frac{K \cdot N_{p}}{F_{1}} \\
& r_{L}=\frac{\alpha \cdot K \cdot N_{L}}{F_{2}}
\end{aligned}
$$

Em que $N_{p}$ e $N_{L}$ são respectivamente, o índice de resistência à penetração na cota de apoio da ponta da estaca e o índice médio de resistência à penetração na camada de solo de espessura $\Delta \mathrm{L}$ (Figura 1).

Assim, no método de Aoki-Velloso, a capacidade de carga ( $R$ ) de um elemento isolado de fundação é estimada pela fórmula semiempírica mostrada na Equação 11.

$$
R=\frac{K \cdot N_{p}}{F_{1}} \cdot A_{p}+\frac{U}{F_{2}} \cdot \sum_{1}^{n}\left(\alpha \cdot K \cdot N_{L} \cdot \Delta_{L}\right) \quad \text { Eq.[11] }
$$

Os valores de $\mathrm{K}$ e de $\alpha$ são expressos na

\begin{tabular}{|c|c|c|}
\hline Solo & $\mathrm{K}(\mathrm{MPa})$ & $\alpha(\%)$ \\
\hline Areia & 1,00 & 1,4 \\
\hline Areia siltosa & 0,80 & 2,0 \\
\hline Areia siltoargilosa & 0,70 & 2,4 \\
\hline Areia argilosa & 0,60 & 3,0 \\
\hline Areia argilossiltosa & 0,50 & 2,8 \\
\hline Silte & 0,40 & 3,0 \\
\hline Silte arenoso & 0,55 & 2,2 \\
\hline Silte arenoargiloso & 0,45 & 2,8 \\
\hline Silte argiloso & 0,23 & 3,4 \\
\hline Silte argiloarenoso & 0,25 & 3,0 \\
\hline Argila & 0,20 & 6,0 \\
\hline Argila arenosa & 0,35 & 2,4 \\
\hline Argila arenossiltosa & 0,30 & 2,8 \\
\hline Argila siltosa & 0,22 & 4,0 \\
\hline Argila siltoarenosa & 0,33 & 3,0 \\
\hline
\end{tabular}
Tabela 2, enquanto os valores de F1 e F2 estão dispostos na Tabela 3.

TABELA 2: Coeficiente K e razão de atrito $\alpha$.

\section{TABELA 3: Fatores de correção F, e F2.}

\begin{tabular}{lcc}
\hline Tipo de estaca & F1 & F2 \\
\hline Franki & 2,50 & 5,00 \\
\hline Metálica & 1,75 & 3,50 \\
\hline Pré-moldada & $1+D / 0,80$ & $2 \cdot F 1$ \\
\hline Escavada & 3,00 & 6,00 \\
\hline Raiz, Hélice Contínua, Ômega & 2,00 & 4,00 \\
\hline
\end{tabular}




\subsection{Método de cálculo Décourt-Quaresma}

O método proposto por Décourt e Quaresma (1978) faz a consideração das parcelas de resistência $\left(R_{L} \quad\right.$ e $\left.\quad R_{p}\right)$, necessárias para a determinação da capacidade de carga em um elemento isolado de fundação, representando-as como mostrado nas equações 12 e 13.

$$
\begin{gathered}
R_{L}=r_{L} \cdot U \cdot L \\
R_{p}=r_{p} \cdot A_{p}
\end{gathered}
$$

O procedimento desenvolvido por esses pesquisadores, faz uma estimativa da tensão de adesão ou de atrito lateral $\left(r_{L}\right)$ calculando o valor médio do índice de resistência à penetração do ensaio de SPT ao longo do fuste $\left(\mathrm{N}_{\mathrm{L}}\right)$ sem fazer qualquer distinção quanto as camadas de solo que compõem a região a ser inserida a estaca. Além disso, esse método limita os valores inferior e superior do $\mathrm{N}_{\mathrm{SPT}}$, de três e quinze respectivamente, além de não considerar os valores que serão utilizados no cálculo da parcela resistida pela ponta. Quando esses cálculos foram propostos em 1978, definiram-se também os valores de entrada para $r_{L}$ e $r_{p}$, contudo em 1982, os valores tabelados existentes foram modificados por Décourt, passando a serem calculados pelas Equações $14 \mathrm{e}$ 15:

$$
\begin{gathered}
r_{L}=10 \cdot\left(\frac{N_{L}}{3}+1\right) \\
r_{p}=C \cdot N_{p}
\end{gathered}
$$

Em que:

$\mathrm{N}_{\mathrm{p}}$ é o valor médio do índice de resistência à penetração na ponta ou base da estaca, obtidos a partir do valor correspondente ao nível da ponta e os valores imediatamente anterior e posterior.

$C$ é coeficiente característico do solo, dispostos na Tabela 4.

TABELA 4: Coeficiente característico do solo.

\begin{tabular}{lc} 
Tipo de solo & C (kPa) \\
\hline Argila & 120 \\
\hline Silte argiloso* & 200 \\
\hline Silte arenoso* & 250 \\
\hline Areia & 400 \\
\hline * Solos residuais & FONTE: Aoki e Cintra (2010).
\end{tabular}

Outra alteração desse método ocorre em 1996 quando Décourt introduz fatores $\alpha$ e $\beta$, respectivamente nas parcelas de resistência de ponta e resistência lateral, resultando na equação 16.

$$
R=\alpha \cdot C \cdot N_{p} \cdot A_{p}+\beta \cdot 10 \cdot\left(\frac{N_{L}}{3}+1\right) \cdot U \cdot L \quad \text { Eq.[16] }
$$

Os valores propostos para $\alpha$ e $\beta$ são em função da composição do solo e do tipo de estaca a ser executada na fundação. As Tabelas 5 e 6 apresentam os valores definidos para esses novos parâmetros.

TABELA 5: Valores do fator $\alpha$ em função do tipo de estaca e do tipo de solo, resistência de ponta.

\begin{tabular}{lccccc}
\hline Tipo de solo & Escavada em geral & Escavada (betonita) & Hélice Continua & Raiz & Injetada \\
\hline Argilas & 0,85 & 0,85 & $0,3^{*}$ & $0,85^{*}$ & $1^{*}$ \\
\hline Solos intermediários & 0,6 & 0,6 & $0,3^{*}$ & $0,6^{*}$ & $1^{*}$ \\
\hline Areias & 0,5 & 0,5 & $0,3^{*}$ & $0,5^{*}$ & $1^{*}$ \\
\hline
\end{tabular}


TABELA 6: Valores do fator $\beta$ em função do tipo de estaca e do tipo de solo, resistência lateral.

\begin{tabular}{|c|c|c|c|c|c|}
\hline Tipo de solo & Escavada em geral & Escavada (betonita) & Hélice Continua & Raiz & Injetada \\
\hline Argilas & $0,8^{*}$ & $0,9 *$ & $1^{*}$ & $1,5^{*}$ & $3^{*}$ \\
\hline Solos intermediários & $0,65^{*}$ & $0,75^{*}$ & $1^{*}$ & $1,5^{*}$ & $3^{*}$ \\
\hline Areias & $0,5^{*}$ & $0,6^{*}$ & $1^{*}$ & $1,5^{*}$ & $3^{*}$ \\
\hline
\end{tabular}

Os valores apresentados com * são apenas orientativos diante do reduzido número de dados disponíveis FONTE: Aoki e Cintra (2010).

Para as estacas pré-moldadas, metálicas e tipo Franki, $\alpha$ e $\beta$ permanecem 1 , como o da proposição inicial.

Observa-se por meio das Tabelas 5 e 6 que há valores de coeficiente apenas orientativos, em função do pequeno número de ensaios. Em função disto, utilizou-se resultados de prova de carga estática de estacas, com o intuito de comparar resultados obtidos pelos modelos analíticos e confrontá-los com os valores experimentais.

\subsection{Método de cálculo de Teixeira}

Teixeira (1996), na tentativa de uma unificação entre os métodos de Aoki-Velloso (1975) e Décourt-Quaresma (1978) propõe que o cálculo da capacidade de carga seja feito utilizando outros parâmetros para $\alpha$ e $\beta$. Assim, por meio da
Equação 1 desenvolve-se a Equação 17.

$R=R_{p}+R_{L}=\alpha \cdot N_{p} \cdot A_{p}+\beta \cdot N_{L} \cdot U \cdot L \quad$ Eq.[17]

Em que:

$\mathrm{N}_{\mathrm{p}}$ é o valor médio do índice de resistência à penetração medido no intervalo de quatro diâmetros acima da ponta e um diâmetro abaixo; $\mathrm{N}_{\mathrm{L}}$ é o valor médio do índice de resistência à penetração ao longo do fuste da estaca.

Os valores proposto por Teixeira para o parâmetro $\alpha$ são decorrentes do solo e do tipo de estaca, e são apresentados na Tabela 7.

Os valores sugeridos para o parâmetro $\beta$ são decorrentes apenas do tipo de estaca, independem da composição das camadas do solo, os mesmos são mostrados na Tabela 8.

TABELA 7: Valores do parâmetro $\alpha$.

\begin{tabular}{|c|c|c|c|c|}
\hline Solo $(4<$ SPT < 40) & Metálica & Escavada & Franki & Raiz \\
\hline Areia & 400 & 270 & 340 & 260 \\
\hline Areia siltosa & 360 & 240 & 300 & 220 \\
\hline Areia argilosa & 300 & 200 & 240 & 190 \\
\hline Silte* & 160 & 110 & 120 & 110 \\
\hline Silte arenoso & 260 & 160 & 210 & 160 \\
\hline Silte argiloso & 160 & 110 & 120 & 110 \\
\hline Argila* & 110 & 100 & 100 & 100 \\
\hline Argila arenosa & 210 & 130 & 160 & 140 \\
\hline Argila siltosa & 110 & 100 & 100 & 100 \\
\hline
\end{tabular}




\section{TABELA 8: Parâmetro $\beta$ ( $\mathrm{kPa})$ (Resistência Lateral).}

\begin{tabular}{cc} 
Tipo de estaca & $\boldsymbol{\beta}(\mathrm{kPa})$ \\
\hline Metálica & 4 \\
\hline Escavada & 4 \\
\hline Franki & 5 \\
\hline Raiz & 6 \\
\hline & Fonte: Aoki e Cintra (2010).
\end{tabular}

\subsection{COMPARAÇÃO ENTRE OS MÉTODOS}

Para uma melhor visualização das diferenças dos métodos, apresenta-se na Tabela 9 uma comparação entre os valores utilizados de $\mathrm{N}_{\mathrm{SPT}}$.

\section{APRESENTAÇÃO DA ROTINA COMPUTACIONAL}

Inicialmente o usuário deverá informar à rotina computacional os parâmetros do ensaio de percussão simples e escolher o tipo de estaca, informando também o diâmetro e a cota de arrasamento da ponta da mesma. Na Figura 2, apresenta-se a primeira etapa da rotina computacional.

Nas próximas etapas, o usuário deverá escolher um método de cálculo e a rotina apresentará os valores da capacidade de carga das estacas de forma automática. Nas Figuras 3, 4 e 5, apresentam-se os resultados dos modelos desenvolvidos por Aoki-Velloso (1975), DécourtQuaresma (1978) e Teixeira (1986), respectivamente.

TABELA 9: Comparação entre os valores utilizados de SPT considerados por cada método.

\begin{tabular}{|c|c|}
\hline \multirow{2}{*}{ Aoki-Velloso (1975) } & $N_{p}=$ Índice $N_{\text {SPT }}$ na cota de apoio da ponta da estaca; \\
\hline & $N_{L}=$ Índice $N_{S P T}$ médio na camada de solo de espessura $\Delta_{\mathrm{L}}$. \\
\hline \multirow{2}{*}{ Décourt-Quaresma (1978) } & $\begin{array}{l}N_{p}=\text { Valor médio do } N_{S P T} \text { na base da estaca, obtido a partir } \\
\text { de } 3 \text { valores: o da ponta, imediatamente anterior e posterior; }\end{array}$ \\
\hline & $N_{L}=$ Índice $N_{\text {SPT }}$ médio ao longo do fuste da estaca. \\
\hline \multirow{2}{*}{ Teixeira (1996) } & $\begin{array}{l}\quad N_{p}=\text { Valor médio do } N_{\mathrm{SPT}} \text { medido no intervalo de } 4 \\
\text { diâmetros acima da ponta da estaca e } 1 \text { diâmetro abaixo; }\end{array}$ \\
\hline & $N_{L}=$ Índice $N_{\mathrm{SPT}}$ médio ao longo do fuste da estaca. \\
\hline
\end{tabular}




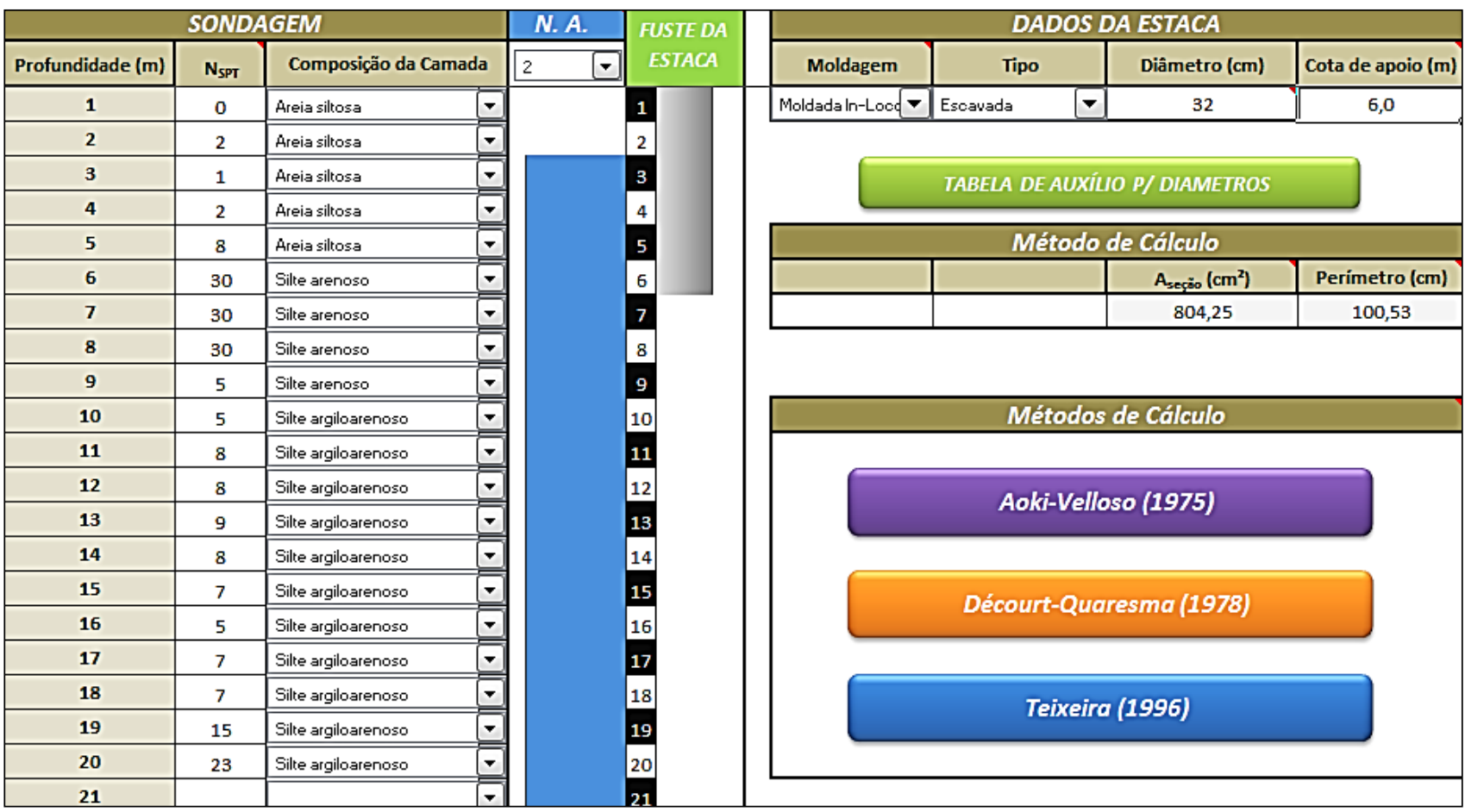

FIGURA 2: Sondagem e informação do tipo de estaca.

Fonte: Próprios autores.

\begin{tabular}{|c|c|c|c|c|c|c|c|c|c|}
\hline \multicolumn{10}{|c|}{ MÉTODO AOKI VELLOSO (1975) } \\
\hline \multicolumn{8}{|c|}{ Dados do Cálculo } & \multirow{2}{*}{\multicolumn{2}{|c|}{ AVANÇAR }} \\
\hline Estaca & \multicolumn{4}{|c|}{ Tіро } & Diâmetro $(\mathrm{cm})$ & Comp. (m) & F.S (Global) & & \\
\hline Moldada in-loco & \multicolumn{4}{|c|}{ Escavada } & 32 & 6 & \multirow[t]{3}{*}{2} & \multirow{3}{*}{\multicolumn{2}{|c|}{ VOLTAR }} \\
\hline \multicolumn{4}{|c|}{ \% Resistência de ponta } & \multicolumn{3}{|c|}{ \% Resistência lateral } & & & \\
\hline \multicolumn{4}{|c|}{100} & \multicolumn{3}{|c|}{100} & & & \\
\hline \multicolumn{10}{|c|}{ RESULTADOS } \\
\hline Profundidade $(\mathrm{m})$ & $\mathrm{K}(\mathrm{kPa})$ & $\alpha$ & F1 & F2 & $R_{P \text { na camada }}(\mathrm{kN})$ & $R_{\text {Lpor metro }}(\mathrm{kN})$ & $\mathbf{R}_{\text {Lacumlada }}(\mathrm{kN})$ & $\mathbf{R}_{\text {Total do solo }}(\mathbf{k N})$ & $P_{\text {Adm }}(k N)$ \\
\hline 1 & 800 & 0,02 & 3 & 6 & 0,00 & 0,00 & 0,00 & 0,00 & 0,00 \\
\hline 2 & 800 & 0,02 & 3 & 6 & 42,89 & 5,36 & 5,36 & 48,25 & 24,13 \\
\hline 3 & 800 & 0,02 & 3 & 6 & 21,45 & 2,68 & 8,04 & 29,49 & 14,74 \\
\hline 4 & 800 & 0,02 & 3 & 6 & 42,89 & 5,36 & 13,40 & 56,30 & 28,15 \\
\hline 5 & 800 & 0,02 & 3 & 6 & 171,57 & 21,45 & 34,85 & 206,42 & 103,21 \\
\hline 6 & 550 & 0,022 & 3 & 6 & 442,34 & 60,82 & 95,67 & 538,01 & 269,00 \\
\hline
\end{tabular}

FIGURA 3: Capacidade de carga, Método Aoki-Velloso (1975).

FONTE: Próprios autores.

\begin{tabular}{|c|c|c|c|c|c|c|c|c|c|c|c|c|}
\hline \multicolumn{13}{|c|}{ MÉTODO DECÓURT QUARESMA (1978) } \\
\hline \multicolumn{11}{|c|}{ DADOS PARA CÁLCULO } & \multirow{3}{*}{\multicolumn{2}{|c|}{ AVANÇAR }} \\
\hline Estaca & \multicolumn{5}{|c|}{ Tipo } & Diâmetro $(\mathrm{cm})$ & $A p\left(m^{2}\right)$ & $U(m)$ & \multicolumn{2}{|c|}{ Comprimento $(\mathrm{m})$} & & \\
\hline Moldada in-loco & \multicolumn{5}{|c|}{ Escavada } & 32 & 0,0804 & 1,005 & \multicolumn{2}{|c|}{6} & & \\
\hline & \multicolumn{9}{|c|}{ AUUSTES DO MÉTODO DÉCOURT-QUARESMA } & & \multicolumn{2}{|c|}{ VOLTAR } \\
\hline & \multicolumn{5}{|c|}{ Coef. Solo $(\mathrm{kPa})$} & \multicolumn{2}{|c|}{ \% Resistência de ponta (a) } & \multicolumn{2}{|c|}{ \% Resistência lateral ( $\beta$ ) } & \multirow{2}{*}{\multicolumn{3}{|c|}{ Parâmetros do Método }} \\
\hline & \multicolumn{5}{|c|}{ Variável de acordo com o Solo* } & \multicolumn{2}{|c|}{100} & \multicolumn{2}{|c|}{0} & & & \\
\hline \multicolumn{11}{|c|}{ RESULTADOS } & \multicolumn{2}{|c|}{ Verificação } \\
\hline Profundidade $(\mathrm{m})$ & $N_{p}$ & $N_{L}$ & $\alpha(\%)$ & $\beta(\%)$ & Coef. solo & $R_{\mathrm{P}}(\mathrm{kN})$ & $R_{L}(k N)$ & $R_{\text {Lacumlada }}(\mathrm{kN})$ & $\mathbf{R}_{\text {Total }}(\mathbf{k N})$ & $P_{\text {Adm }}(k N)$ & $R / 2$ & $(R I / 1,3)+(R p / 4)$ \\
\hline 1 & 1,00 & 3,00 & 0,60 & 0,65 & 400,00 & 19,30 & 0,00 & 0,00 & 19,30 & 4,83 & 9,65 & 4,83 \\
\hline 2 & 1,00 & 3,00 & 0,60 & 0,65 & 400,00 & 19,30 & 0,00 & 0,00 & 19,30 & 4,83 & 9,65 & 4,83 \\
\hline 3 & 1,67 & 3,00 & 0,60 & 0,65 & 400,00 & 32,17 & 0,00 & 0,00 & 32,17 & 8,04 & 16,08 & 8,04 \\
\hline 4 & 3,67 & 3,00 & 0,60 & 0,65 & 400,00 & 70,77 & 0,00 & 0,00 & 70,77 & 17,69 & 35,39 & 17,69 \\
\hline 5 & 13,33 & 4,00 & 0,60 & 0,65 & 400,00 & 257,36 & 0,00 & 0,00 & 257,36 & 64,34 & 128,68 & 64,34 \\
\hline 6 & 22,67 & 5,83 & 0,60 & 0,65 & 250,00 & 273,44 & 0,00 & 0,00 & 273,44 & 68,36 & 136,72 & 68,36 \\
\hline
\end{tabular}

FIGURA 4: Capacidade de carga, Método Decóurt-Quaresma (1979).

FONTE: Próprios autores. 


\begin{tabular}{|c|c|c|c|c|c|c|}
\hline \multicolumn{7}{|c|}{ MÉTODO TEIXEIRA (1996) } \\
\hline \multicolumn{7}{|c|}{ DADOS PARA CÁLCULO } \\
\hline Estaca & Tipo & Diâmetro $(\mathrm{cm})$ & $A p\left(m^{2}\right)$ & $U(m)$ & $\operatorname{Comp}(\mathrm{m})$ & F. Segurança \\
\hline Moldada in-loco & Escavada & 32 & 0,0804 & 1,005 & 6 & 2 \\
\hline \multicolumn{5}{|c|}{ \% Resistência de ponta } & \multicolumn{2}{|c|}{ \% Resistência lateral } \\
\hline & & & \multicolumn{2}{|l|}{100} & \multicolumn{2}{|c|}{0} \\
\hline
\end{tabular}

\begin{tabular}{c|c|c|c|c|c|c|c|c|c|c|}
\hline \multicolumn{10}{|c|}{ RESULTADOS } \\
\hline Profundidade $(\mathbf{m})$ & $\boldsymbol{N}_{\boldsymbol{p}}$ & $\boldsymbol{N}_{\boldsymbol{L}}$ & $\boldsymbol{\alpha}(\mathbf{k P a})$ & $\boldsymbol{\beta}(\mathbf{k P a})$ & $\mathbf{R}_{\mathbf{p}}(\mathbf{k N})$ & $\mathbf{R}_{\mathbf{L}}(\mathbf{k N})$ & $\mathbf{R}_{\text {Lacumlada }}(\mathbf{k N})$ & $\mathbf{R}_{\text {Total }}(\mathbf{k N})$ & $\mathbf{P}_{\text {Adm }}(\mathbf{k N})$ \\
\hline $\mathbf{1}$ & 4,00 & 4,00 & 240 & 4 & 77,21 & 0,00 & 0,00 & 77,21 & $\mathbf{3 8 , 6 0}$ \\
\hline $\mathbf{2}$ & 4,00 & 4,00 & 240 & 4 & 77,21 & 0,00 & 0,00 & 77,21 & 38,60 \\
\hline $\mathbf{3}$ & 4,00 & 4,00 & 240 & 4 & 77,21 & 0,00 & 0,00 & 77,21 & 38,60 \\
\hline $\mathbf{4}$ & 4,00 & 4,00 & 240 & 4 & 77,21 & 0,00 & 0,00 & 77,21 & 38,60 \\
\hline $\mathbf{5}$ & 6,00 & 4,80 & 240 & 4 & 115,81 & 0,00 & 0,00 & 115,81 & 57,91 \\
\hline $\mathbf{6}$ & 19,00 & 9,00 & 160 & 4 & 244,49 & 0,00 & 0,00 & 244,49 & 122,25 \\
\hline
\end{tabular}

FIGURA 5: Capacidade de carga, Método Teixeira (1996).

FONTE: Próprios autores.

Por fim, apresenta-se um resumo dos três métodos de cálculo para a determinação da capacidade de carga de estacas. Na Figura 6, apresenta-se um gráfico que representa a capacidade de carga das estacas ao longo da profundidade do fuste.
No sítio eletrônico do curso de Engenharia Civil (www.engicivilufgcac.com.br) do Campus Catalão, é possível "descarregar" a rotina computacional para o cálculo da capacidade de carga em estacas.

\begin{tabular}{|c|c|c|c|c|}
\hline \multicolumn{5}{|c|}{ RELATÓRIO FINAL } \\
\hline Estaca & \multicolumn{2}{|c|}{ Tipo } & Diâmetro $(\mathrm{cm})$ & $N . A(m)$ \\
\hline Pré-Moldada & \multicolumn{2}{|c|}{ Vibrada Concreto } & 25 & 2 \\
\hline \multicolumn{5}{|c|}{ Carga Admissivel na cota de apoio da Estca (kN) } \\
\hline Cota $(m)$ & Aoki-Velloso & Décourt-Quaresma & Teixeira & Média \\
\hline 6,00 & 393,97 & 125,59 & 168,06 & 229,21 \\
\hline \multicolumn{5}{|c|}{ Carga Geotécnica Admissível (kN) } \\
\hline Profundidade (m) & Aoki-Velloso & Décourt-Quaresma & Teixeira & Média \\
\hline 0 & 0 & 0 & 0 & 0 \\
\hline 1 & 0,00 & 4,91 & 41,63 & 15,51 \\
\hline 2 & 34,71 & 4,91 & 47,91 & 29,18 \\
\hline 3 & 22,14 & 20,26 & 54,19 & 32,20 \\
\hline 4 & 41,89 & 42,16 & 60,48 & 48,18 \\
\hline 5 & 150,80 & 103,71 & 85,69 & 113,40 \\
\hline 6 & 393,97 & 125,59 & 168,06 & 229,21 \\
\hline
\end{tabular}

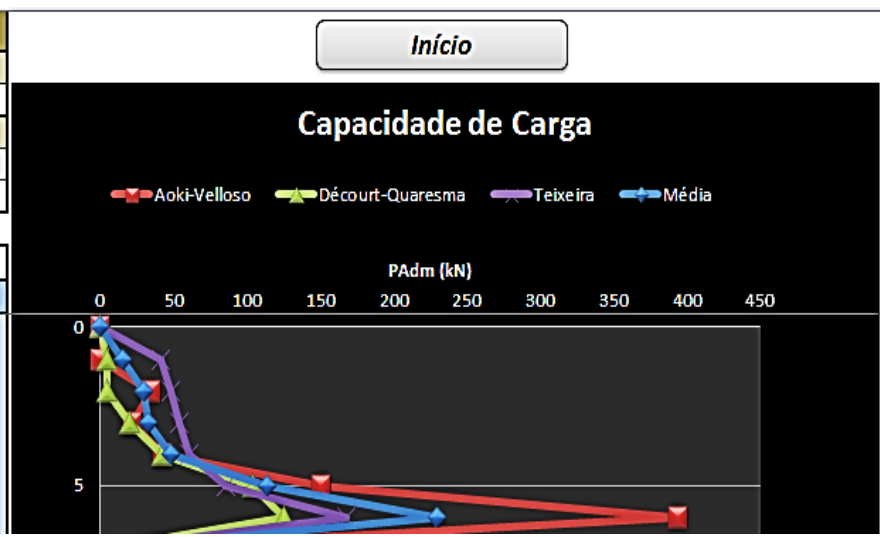

FIGURA 6: Resumo das capacidades de carga de estacas.

FONTE: Próprios autores. 


\section{VALORES DE AFERIÇÃO}

O desenvolvimento da rotina computacional para o cálculo da capacidade de carga de estacas foi baseada em métodos aplicáveis para solos com características específicas. Faz-se necessário que os coeficientes sejam ajustados para os solos das diferentes regiões do país. Com o objetivo de avaliar os resultados da rotina computacional e propor pesquisas para a obtenção de parâmetros de correção para os solos da região Centro-Oeste, utilizaram-se valores de três provas de cargas, duas executadas em Brasília-DF e uma de Goiânia-GO. Os resultados das provas de carga foram obtidos em Magalhães (2005).

O primeiro ensaio de prova de carga é designado por PC-01BR, o qual apresenta uma curva carga-recalque definida. Trata-se de uma prova de carga em estaca curta (L/D $=25)$ de $35 \mathrm{~cm}$ de diâmetro, tipo Hélice contínua monitorada. A prova de carga foi interrompida com carga última evidenciada, provocando recalque superior a 30 $\mathrm{mm}$, ou seja, superior à deformação limite prevista pela NBR 6122:2010 e deformação relativa maior que $8,5 \%$ do diâmetro da estaca na carga máxima atingida pelo teste. A estaca tem comprimento 8,60 $\mathrm{m}$, carga última de ensaio igual a $1400 \mathrm{KN}$. No Quadro 1 é mostrado o boletim de sondagem a percussão simples do solo onde foi realizada a prova de carga da estaca PC-01BR.

O segundo ensaio de prova de carga utilizado foi para a estaca PC-01BR, a qual apresenta uma curva carga-recalque definida. Trata-se de uma prova de carga em estaca intermediária $(L / D=46)$ de $40 \mathrm{~cm}$ de diâmetro. $O$ ensaio foi interrompido com carga máxima evidenciada e com valor de recalque superior a deformação limite prevista pela NBR-6122:2010 e deformação relativa próxima de $6 \%$ do diâmetro da estaca em relação a carga máxima atingida pelo ensaio. A referida estaca tem comprimento igual a 18,50 m e apresentou carga máxima de ensaio igual a 1100 kN. No Quadro 2 apresenta-se o boletim de sondagem a percussão simples do solo onde foi realizada a prova de carga da estaca PC-02BR.

A terceira prova de carga utilizada foi para a estaca PC-02GO, que também apresenta uma curva carga-recalque definida. Trata-se de uma prova de carga em estaca intermediária (L/D = 37) de $40 \mathrm{~cm}$ de diâmetro, comprimento igual a $15 \mathrm{~m}$ e foi interrompida com recalque próximo de $10 \mathrm{~mm}$, resultando numa deformação relativa próxima de $3 \%$ do diâmetro da estaca em relação à carga máxima do ensaio, cujo valor foi igual a $1200 \mathrm{kN}$. Por meio do Quadro 3 mostra-se o boletim de sondagem a percussão simples do solo onde foi realizada a prova de carga da estaca PC-02GO.

QUADRO 1: Sondagem tipo SPT - PC-01BR.

\begin{tabular}{|c|c|c|}
\hline Prof. (m) & $\mathbf{N}_{\text {SPT }}$ & Tipo de solo \\
\hline $0-1$ & 4 & \multirow{2}{*}{$\begin{array}{c}\text { Argila pouco siltosa com } \\
\text { pedregulhos, mole, vermelha }\end{array}$} \\
\hline $1-2$ & 11 & \\
\hline $2-3$ & 16 & \multirow{12}{*}{$\begin{array}{l}\text { Silte pouco argiloso, rijo a } \\
\text { duro, variegado }\end{array}$} \\
\hline $3-4$ & $37 / 15$ & \\
\hline $4-5$ & 24 & \\
\hline $5-6$ & 45 & \\
\hline $6-7$ & - & \\
\hline $7-8$ & - & \\
\hline $8-9$ & - & \\
\hline $9-10$ & 35 & \\
\hline $10-11$ & - & \\
\hline $11-12$ & - & \\
\hline $12-13$ & $25 / 7$ & \\
\hline $13-14$ & - & \\
\hline
\end{tabular}

FONTE: Magalhães (2005).

QUADRO 2: Sondagem tipo SPT - PC-02BR.

\begin{tabular}{|c|c|c|}
\hline Prof. $(\mathbf{m})$ & $\mathbf{N}_{\text {SPT }}$ & \multirow{2}{*}{ Tipo de solo } \\
\hline $0-1$ & 6 & \multirow{3}{*}{ Aterro Consolidado } \\
\hline $1-2$ & 6 & \\
\hline $2-3$ & 10 & \\
\hline $3-4$ & 10 & \\
\hline $4-5$ & 6 & \\
\hline $5-6$ & 2 & \\
\hline $6-7$ & 2 \\
\hline $7-8$ & 6 & \\
\hline $8-9$ & 6 & \\
\hline $9-10$ & 10 \\
\hline $10-11$ & 12 \\
\hline $11-12$ & 12 \\
\hline $12-13$ & 14 \\
\hline $13-14$ & 26 \\
\hline $14-15$ & 9 \\
\hline $15-16$ & 10 \\
\hline $16-17$ & 12 \\
\hline $17-18$ & 39 & \\
\hline $18-19$ & 49 & \\
\hline $19-20$ & 50 & \\
\hline & \\
\end{tabular}

FONTE: Magalhães (2005). 


\section{QUADRO 3: Sondagem tipo SPT - PC-02GO.}

\begin{tabular}{|c|c|c|}
\hline Prof. (m) & $\mathbf{N}_{\text {SPT }}$ & \multirow{2}{*}{ Tipo de solo } \\
\hline $0-1$ & 8 & \multirow{2}{*}{ Silte arenoso, rósea } \\
\hline $1-2$ & 12 & \\
\hline $2-3$ & 13 & \\
\hline $3-4$ & 13 & \multirow{2}{*}{ Silte arenoso, rósea e amarelo } \\
\hline $4-5$ & 13 & \\
\hline $5-6$ & 13 & \\
\hline $6-7$ & 15 & \\
\hline $7-8$ & 14 & \\
\hline $8-9$ & 18 & \\
\hline $9-10$ & 19 & \multirow{2}{*}{ Silte arenoso, micáceo } \\
\hline $10-11$ & 19 & \\
\hline $11-12$ & 20 & \\
\hline $12-13$ & 21 & \\
\hline $13-14$ & 28 \\
\hline $14-15$ & 28 & \\
\hline $15-16$ & 48 & \\
\hline $16-17$ & 50 & \\
\hline & &
\end{tabular}

FONTE: Magalhães (2005).

\section{RESULTADOS E DISCUSSÃO}

$\mathrm{Na}$ Tabela 10, apresentam-se os resultados obtidos por meio dos métodos AokiVelloso (1975), Décourt-Quaresma (1978) e Teixeira (1996). É importante ressaltar que os valores obtidos pelos métodos semiempíricos, não foram afetadas pelos coeficientes de seguranças externos.

Observa-se por meio da Tabela 10, que os coeficientes de correção utilizados pelos métodos semiempíricos, necessitam de ajustes para solos de cada região do país. Verifica-se que o método de Aoki-Velloso (1975), apresentou valores contra a segurança, quando comparados com os valores experimentais. Os resultados dos modelos de Décourt-Quaresma (1978) e Teixeira (1996) apresentaram resultados conservadores.

Para o método de Aoki-Velloso (1975), para as provas de carga PC01BR e PC02BR, indicamse que os coeficientes $\alpha$ para argila, argila siltosa e silte tenham os respectivos valores: 0,56, 0,035 e 0,026 . Para os valores do coeficiente $K$, sugerem-se $178 \mathrm{kPa}$ para argilas, $193 \mathrm{kPa}$ para argila siltosa e 350 para siltes. Para a prova de carga PC02GO, os valores de $\alpha$ para silte arenoso tenha o valor de 0,0136 e K $=341 \mathrm{kPa}$.

Para o modelo de Décourt-Quaresma, nos provas de carga PC01BR e PC02BR sugerem-se que os coeficientes $C$ tenham os valores de $268 \mathrm{kPa}$ e $558 \mathrm{kPa}$ para argila siltosa e silte respectivamente. Para a prova de carga PC02GO o valor do coeficiente $C$ não necessita de ajuste.

Para o método de Teixeira (1996), para as provas de carga PC01BR e PC02BR, indicam-se que os coeficientes $\alpha$ para argila, argila siltosa e silte tenham os respectivos valores: $143 \mathrm{kPa}, 157 \mathrm{kPa}$ e $254 \mathrm{kPa}$. Para os valores do coeficiente $\beta$, sugeremse $5,72 \mathrm{kPa}$ para argilas e argila siltosa e 4,16 para siltes. Para a prova de carga $\mathrm{PCO} 2 \mathrm{GO}$, os valores de $\alpha$ para silte arenoso tenha o valor de $125 \mathrm{kPa}$ e $\beta=3,12 \mathrm{kPa}$.

TABELA 10: Comparação entre as provas de cargas e os métodos da rotina computacional.

\begin{tabular}{|c|c|c|c|c|c|c|c|c|c|c|}
\hline \multirow{2}{*}{ Estaca } & \multirow{2}{*}{$\begin{array}{c}\begin{array}{c}\text { Prova de } \\
\text { Carga }\end{array} \\
\mathbf{P}_{\text {exp }}\end{array}$} & \multicolumn{3}{|c|}{ Aoki-Velloso } & \multicolumn{3}{|c|}{ Décourt-Quaresma } & \multicolumn{3}{|c|}{ Teixeira } \\
\hline & & $\mathbf{P}_{\text {rup }}$ & $\mathbf{P}_{\mathrm{adm}}$ & $\mathbf{P}_{\text {exp }} / \mathbf{P}_{\text {rup }}$ & $P_{\text {rup }}$ & $\mathbf{P}_{\mathrm{adm}}$ & $\mathbf{P}_{\text {exp }} / \mathbf{P}_{\text {rup }}$ & $\mathbf{P}_{\text {rup }}$ & $\mathbf{P}_{\mathrm{adm}}$ & $P_{\text {exp }} / P_{\text {rup }}$ \\
\hline PC01BR & 1400 & 1600 & 800 & 0,88 & 630 & 315 & 2,22 & 982 & 491 & 1,43 \\
\hline PC02BR & 1100 & 1236 & 618 & 0,81 & 860 & 430 & 1,27 & 1026 & 513 & 1,07 \\
\hline PC02GO & 1200 & 1933 & 966 & 0,62 & 1181 & 590 & 1,02 & 1536 & 768 & 0,78 \\
\hline
\end{tabular}

$\mathrm{P}_{\exp }$ representa o valor da carga de ruptura experimental, obtida por meio dos ensaios de prova de carga.

$P_{\text {rup }}$ é a capacidade de carga de ruptura da estaca, obtida por meio dos métodos semi-empíricos.

$\mathrm{P}_{\mathrm{adm}}$ é a capacidade de carga admissível da estaca, obtida por meio dos métodos semi-empíricos. 


\section{CONSIDERAÇÕES FINAIS}

Os resultados apresentados pela rotina computacional mostraram-se eficientes para uma análise comparativa entre os três métodos de cálculo, ficando evidente que em decorrência das considerações feitas por cada método, o resultado final apresenta variação. Tais variações devem ser analisadas com rigor pelo engenheiro no ato de especificar o tipo de fundação a ser utilizado.

Destaca-se que a formulação desenvolvida neste artigo, é apenas uma das diversas soluções possíveis para a previsão de carga de uma fundação em estaca, além disso, às variações de cada tipo de solo e recursos para a execução das fundações, aumentam consideravelmente as possibilidades de pesquisa nesta área.

Constata-se que é necessária, uma ampla pesquisa com a execução de provas de carga em todas as regiões do país, com intuído de aferir os coeficientes de correção utilizados nos métodos de cálculo analisados neste artigo.

A rotina computacional desenvolvida neste trabalho está disponível no sítio eletrônico do curso de Engenharia Civil do campus Catalão da Universidade Federal de Goiás e tem como objetivo auxilar profissionais habilitados no desenvolvimento de projeto de fundações.

\section{AGRADECIMENTOS}

Ao Conselho de desenvolvimento científico e tecnológico - CNPq, pela bolsa de iniciação científica disponibilizada ao primeiro autor.

\section{REFERÊNCIAS BIBLIOGRÁFICAS}

ALONSO, U. R.. Exercícios de fundação. Editora Edgard Blucher LTDA. São Paulo. p. 71-130. 1983.

ALONSO, Urbano Rodriguez. Previsão e Controle das Fundações. Vol 3. São Paulo. Editora: Edgard Blucher, 1998.

AOKI, N.; VELLOSO, D. A. An proximate method to estimative the bearing capacity of pile. In: Pan American V - P.C.S.M.F.E., Buenos Aires, Proccedings. Vol. 1, p. 367 $-376.1979$.
ASSOCIAÇÃO BRASILEIRA DE NORMAS TÉCNICAS. NBR 6118 - Projeto de estruturas de concreto Procedimento. Rio de Janeiro. 2003.

ASSOCIAÇÃO BRASILEIRA DE NORMAS TÉCNICAS. NBR 6122 - Projeto e Execução de Fundações. Rio de Janeiro. 1994.

CINTRA, J. C. A.; AOKI, N. (2010). Fundações por Estacas: projeto geotécnico. Editora Oficina de Textos, São Paulo, 96 p. 2010.

DÉCOURT, L.; QUARESMA, A. R. Capacidade de carga de estacas a partir de valores de SPT. In: VI COBRAMSEF Rio de Janeiro, 1978.

MAgAlHAES, P. H. L. Avaliação dos métodos de capacidade de carga e recalque de estacas hélice contínua via provas de carga. Dissertação, Universidade de Brasília, Brasília, 2005.

TEIXEIRA, Alberto Henriques (1996). Projeto de execução de fundações. SEFE, 3, São Paulo, vol. 1. 1996. 